\title{
Statin dose in primary prevention: aim for the target!
}

\author{
Márcio S Bittencourt, ${ }^{\oplus 1,2}$ Fernando H Y Cesena $^{3}$
}

Benefits from statin therapy for the primary prevention of atherosclerotic cardiovascular disease (ASCVD) are supported by high-quality evidence from randomised controlled trials (RCT). However, when and how to prescribe statins to individuals without ASCVD is a matter of debate. Strategies for initial management of low-density lipoprotein cholesterol (LDL-c) and goals of treatment differ among different guidelines (table 1). ${ }^{1-3}$ The National Institute for Health and Care Excellence (NICE) guidelines, for instance, recommend daily use of atorvastatin $20 \mathrm{mg}$ in the primary care scenario when the estimated 10-year risk of ASCVD, as assessed by QRISK2, is at least $10 \%$, whereas other guidelines propose different risk equations and different cut-offs. Also, the NICE document recommends clinicians to aim at lowering non-high-density lipoprotein cholesterol (non-HDL-c) by at least $40 \%,{ }^{2}$ whereas other societies propose different eligibility criteria and different targets.

An important step after a guideline publication is the assessment of its uptake among health practitioners and patients in the real world, as well as of the impact of its adherence on clinical outcomes. These analyses may not only verify its appropriateness, providing feedback for continuous improvement of recommendations but also identify targets to optimise delivery of health to the society.

In this context, a study by Akyea et al investigating whether a suboptimal LDL-c response to statins, based on the NICE guidelines, increases the risk of future ASCVD events. Using a large UK prospective cohort of subjects in primary prevention who were prescribed statins, the authors reported that those whose LDL-c lowered by $<40 \%$ within 24 months (over half of the population study) had a higher risk of ASCVD events during a median follow-up of 6.2 years. ${ }^{4}$

\footnotetext{
'Division of Internal Medicine, University Hospital, Sao Paulo, Sao Paulo, Brazil

${ }^{2}$ Department of Internal Medicine, Brigham and Women's Hospital, Boston, Massachusetts, USA ${ }^{3}$ Hospital Israelita Albert Einstein, São Paulo, Sao Paulo, Brazil
}

Correspondence to Dr Márcio S Bittencourt, Division of Internal Medicine, University Hospital, Sao Paulo 2565, Brazil; msbittencourt@mail.harvard.edu
This study allows important insights to those who deal with cholesterol management in primary care. First, the high rate of suboptimal LDL-c response is clearly alarming, although it is probably overestimated for the present day, once the studied cohort was not contemporary (over 40\% of the subjects were recruited before 2006 and managed under older guidelines). Even so, a substantial proportion of individuals who do not attain LDL-c targets recommended by guidelines, especially in high-risk conditions, has already been reported by other investigators. This phenomenon occurs worldwide, irrespective the guideline used by the physicians. ${ }^{5}$

Second, the association of a suboptimal LDL-c response to statin therapy with a higher risk of ASCVD outcomes, as reported, is consistent with RCT showing better outcomes proportioned by more intensive statin therapy, compared with less intensive regimens, although these trials involved mostly individuals in secondary prevention. Moreover, the results fit well with the ample evidence from prospective cohort studies and Mendelian randomisation studies favouring 'the lower the LDL-c, the better' concept, even in primary prevention.

The Heart study by Akyea et al has known limitations inherent to cohort studies, such as the possibility of bias due to unmeasured confounders, although the authors accounted for several potential confounders in the analyses (eg, year of statin initiation, frequency of LDL-c measurements, change of statin potency within the exposure period and deprivation status). Some variables had a huge rate of missing data (eg, unknown status for smoking was reported in $96 \%$ of the cases, and body mass index was not available in $45 \%$ of the subjects). Also, there is no information on actual LDL-c levels during the follow-up, nor adherence to statin therapy was described. Nevertheless, these weaknesses cannot dissipate a clear implication of the study: it is mandatory to understand the reasons for a lower than desirable LDL-c response to statins, in order to propose strategies to improve adherence to guidelines and, as a result, clinical outcomes.

Suboptimal LDL-c response to statins may involve both physician-related and patient-related issues. Physicians may prefer to prescribe low potent statins for several reasons, including unawareness of guideline recommendation, non-convincement of better outcomes with higher potent statins or when a lower LDL-c is attained, and fear of adverse reactions or drug interactions. Moreover, doctors may be reluctant to up-titrate drugs when the treatment goals are not achieved, the so-called therapeutic inertia.

In the Akyea et al study, not surprisingly, optimal responders, as compared with non-optimal responders, were initially prescribed medium potent statins more frequently and low potent statins less often. This probably explains why baseline LDL-c was higher in optimal responders, indicating that higher LDL-c motivates the physician to be more aggressive upfront. ${ }^{4}$

There are also different patient-related causes for a suboptimal LDL-c response to statins. Although an interindividual response to statins may occur according to the genetic background, most cases where LDL-c response is less than expected are probably due to lack of adherence or persistence to the treatment. Even though statin-associated side effects do prevent statin use in many patients, the nocebo effect, that is, the report of a side effect by the patient actually due to negative expectation, is a frequent cause for interrupting statins among the population. Of note, poor adherence to lipid-lowering therapy, together with low-intensity therapy (as opposed to high-intensity treatment), is associated with higher cardiovascular risk. In addition, continued statin use after an adverse reaction has been shown to be related to a lower incidence of death and ASCVD events.

Important issues on blood cholesterol management in primary prevention could not be addressed or answered by the Akyea et al study. NICE recommendation (>40\% non-HDL-c reduction for everyone in primary prevention eligible for statins) is practical and facilitates guideline uptake by clinicians. However, the absolute benefit from statins depends on the baseline LDL-c and ASCVD risk. ${ }^{6}$ There may be subgroups (lower risk and/ or lower LDL-c) where a higher intensive statin (eg, >40\% LDL-c reduction) would provide an incremental absolute benefit of small or negligible magnitude, as compared with a less intensive statin (eg, 30\% LDL-c reduction). On the other hand, there may be subgroups (higher risk and/or higher LDL-c) where an even higher intensive statin therapy (eg, >50\%LDL-c reduction) would prevent significantly more events as compared with a $40 \%$ LDL-c 
Table 1 Recommendations for statin initiation in primary prevention of cardiovascular diseases and goals of treatment, according to different guidelines

\begin{tabular}{|c|c|c|c|}
\hline Guideline & $\begin{array}{l}\text { Score for } \\
\text { cardiovascular } \\
\text { risk assessment }\end{array}$ & Statin eligibility and initial management* & Goal of treatment \\
\hline $\begin{array}{l}\text { NICE (last update: } \\
\text { 2016)2 }\end{array}$ & QRISK2† & Atorvastatin $20 \mathrm{mg}$ per day if risk $\geq 10 \%$ & $\downarrow$ Non-HDL-c by $40 \%$ \\
\hline $\operatorname{ESC}(2016)^{1}$ & SCORE & $\begin{array}{l}\text { Statin considered or recommended as a function of cardiovascular } \\
\text { risk and LDL-c level }\end{array}$ & $\begin{array}{l}\text { Very high risk: LDL-c goal }<1.8 \mathrm{mmol} / \mathrm{L} \text { or } \downarrow \text { LDL-c by } \geq 50 \% \text { if } \\
\text { baseline between } 1.8 \text { and } 3.5 \mathrm{mmol} / \mathrm{L} \\
\text { High risk: } \mathrm{LDL}-\mathrm{c} \text { goal }<2.6 \mathrm{mmol} / \mathrm{L} \text { or } \downarrow \mathrm{LDL}-\mathrm{c} \text { by } \geq 50 \% \text { if } \\
\text { baseline between } 2.6 \text { and } 5.2 \mathrm{mmol} / \mathrm{L} \\
\text { Low or moderate risk: consider LDL-c goal }<3.0 \mathrm{mmol} / \mathrm{L}\end{array}$ \\
\hline $\begin{array}{l}\text { ACC/AHA/NLA/other } \\
(2018)^{3}\end{array}$ & $\begin{array}{l}\text { Pooled cohort } \\
\text { equations }\end{array}$ & $\begin{array}{l}\text { Statin considered or recommended for individuals at borderline } \\
\text { risk ( } 5 \% \text { to }<7.5 \%) \text {, intermediate risk ( } \geq 7.5 \% \text { to }<20 \%) \text {, and high } \\
\text { risk }(\geq 20 \%) \neq \text {. } \\
\text { Risk-enhancing factors§ and coronary artery calcium score may } \\
\text { help decision } \\
\text { If decided for statin in intermediate-risk or borderline-risk patients: } \\
\text { start a moderate-intensity statin }\end{array}$ & $\begin{array}{l}\text { Intermediate-risk: } \downarrow \text { LDL-c by } \geq 30 \% \\
\text { For optimal ASCVD risk reduction, especially in high-risk } \\
\text { patients: } \downarrow \text { LDL-c by } \geq 50 \% \\
\text { Risk-enhancing factors favour intensification of statin therapy } \\
\text { in intermediate-risk patients }\end{array}$ \\
\hline
\end{tabular}

*Decision on statin initiation should be preceded by a discussion with the patient on risks and benefits of the treatment. tQRISK2 should not be used in individuals with an estimated glomerular filtration rate (eGFR) $<60 \mathrm{~mL} / \mathrm{min} / 1.73 \mathrm{~m}^{2}$ and/or albuminuria.

‡Recommendation for individuals aged 40-75 years, with LDL-c levels $1.7-4.8 \mathrm{mmol} / \mathrm{L}$, without ASCVD or diabetes mellitus.

§Risk-enhancing factors include family history of premature ASCVD; primary hypercholesterolaemia (LDL-c 4.1-4.8 mmol/L, non-HDL-c 4.9-5.6 mmol/L); metabolic syndrome; chronic kidney disease; chronic inflammatory conditions; history of premature menopause (before 40 years) or pre-eclampsia; high-risk race/ethnicities; persistently elevated, primary hypertriglyceridaemia ( $\geq 1.98 \mathrm{mmol} / \mathrm{L}$ ); high-sensitivity C reactive protein $\geq 2.0 \mathrm{mg} / \mathrm{L}$; lipoprotein $(\mathrm{a}) \geq 50 \mathrm{mg} / \mathrm{dL}$ or $\geq 125 \mathrm{nmol} / \mathrm{L}$; apolipoprotein $B \geq 130 \mathrm{mg} / \mathrm{dL}$; and anklebrachial index $<0.9$.

ACC, American College of Cardiology; AHA, American Heart Association; ASCVD, atherosclerotic cardiovascular disease; ESC, European Society of Cardiology; HDL-c, high-density lipoprotein cholesterol; LDL-c, low-density lipoprotein cholesterol; NICE, National Institute for Health and Care Excellence; NLA, National Lipid Association; SCORE, Systemic Coronary Risk Estimation.

lowering. For example, a recent meta-analysis demonstrated that more intensive LDL-c-lowering therapy, compared with less potent regimens, is associated with greater reduction in total and cardiovascular mortality when baseline LDL-c is $>2.6 \mathrm{mmol} / \mathrm{L}$, but not when LDL-c is lower. $^{7}$ Therefore, clinicians should use judgement to refine statin therapy beyond general guideline recommendations on a case-by-case basis in their practice, though one should always consider the incremental benefit of more aggressive statin use.

Another point that should deserve further investigation is the comparative analyses of the effectiveness of different guidelines on statin use for the primary prevention of ASCVD. As shown in table 1, criteria for initiate statin therapy and the goals of treatment vary according to the guideline used. These differences are not subtle and may have relevant clinical implications in ASCVD prevention. In this regard, a modelling study from The Copenhagen General Population Study concluded that more events could be prevented by recommending more widespread use of statins compared with more restrictive guidelines. ${ }^{8}$

In conclusion, the study by Akyea et al brings a clear message for both clinicians and patients, highlighting the importance of LDL-c lowering for primary prevention of ASCVD, specifically supporting the concept that not achieving NICE guideline recommendation is a risk factor for ASCVD.

Effective implementation of guidelines among healthcare practitioners and the general population has been a challenge for a long time. Both physicians and patients should be targets for approaches aiming at improving adherence to guidelines. For the clinicians, these strategies should include serious programmes of continuing medical education and the development of in-house protocols. Simplified recommendations and algorithms may also facilitate guideline implementation among physicians. Patients and society should be educated on the scientific evidence documenting the benefits of lipid-lowering therapy and antistatin propaganda based on pseudoscience should be strongly disavowed and demystified by health authorities. Reassurance of statin safety should be emphasised to both doctors and patients in order to diminish excessive, unrealistic concerns. Global efforts and multidirectional strategies are necessary to optimise the management of blood cholesterol in primary care and reduce the burden of ASCVD.

Contributors Both authors have worked on the design, draft and revision of the manuscript.
Funding The authors have not declared a specific grant for this research from any funding agency in the public, commercial or not-for-profit sectors.

Competing interests None declared.

Provenance and peer review Commissioned; internally peer reviewed.

(c) Author(s) (or their employer(s)) 2019. No commercial re-use. See rights and permissions. Published by BMJ.

\section{Check for updates}

To cite Bittencourt MS, Cesena FHY. Heart 2019;105:969-971.

Published Online First 15 April 2019

\section{(2) Linked}

- http://dx.doi.org/10.1136/heartjnl-2018-314253

Heart 2019;105:969-971.

doi:10.1136/heartjnl-2019-314723

\section{REFERENCES}

1 Catapano AL, Graham I, De Backer G, et al. 2016 ESCl EAS Guidelines for the Management of Dyslipidaemias. Eur Heart J 2016;37:2999-3058.

2 Excellence $\mathrm{NIfHaC}$. Cardiovascular disease: risk assessment and reduction, including lipid modification. London: National Institute for Health and Care Excellence, 2016

3 Grundy SM, Stone NJ, Bailey AL, et al. 2018 AHA/ACC/AACVPR/AAPA/ABC/ACPM/ADA/ AGS/APhA/ASPC/NLA/PCNA Guideline on the Management of Blood Cholesterol. Circulation 2018:CIR0000000000000625.

4 Akyea R, Kai J, Qureshi N, et al. Sub-optimal cholesterol response to initiation of statins 
and future risk of cardiovascular disease. Heart 2019; 105:975-81.

5 Danchin N, Almahmeed W, Al-Rasadi K, et al. Achievement of low-density lipoprotein cholesterol goals in 18 countries outside Western Europe: The International ChoLesterol management Practice Study (ICLPS). Eur J Prev Cardiol 2018;25:1087-94.
6 Soran H, Schofield JD, Durrington PN. Cholesterol, not just cardiovascular risk, is important in deciding who should receive statin treatment. Eur Heart $J$ 2015;18:ehv340-ehv.

7 Navarese EP, Robinson JG, Kowalewski M, et al. Association Between Baseline LDL-C Level and Total and Cardiovascular Mortality After LDL-C Lowering: a systematic review and meta-analysis. JAMA 2018:319:1566-79.

8 Mortensen MB, Nordestgaard BG. Comparison of Five Major Guidelines for Statin Use in Primary Prevention in a Contemporary General Population. Ann Intern Med 2018;168:85-92. 